Running Head: RELATIONSHIPS AND REJECTION SENSITIVITY

Rejection Sensitivity in Childhood and Early Adolescence: Peer Rejection and Protective Effects of Parents and Friends 


\begin{abstract}
Theory suggests that rejection sensitivity, a social cognitive processing style characterised by anxious and angry expectations of rejection, develops from experiences of rejection or acceptance by others. The purpose of this study of 417 children and early adolescents (age 9 to 13) was to examine how relationship experiences are directly and interactively associated with their rejection sensitivity. In a multivariate analysis, there was an association of rejection by parents and by peers with rejection sensitivity, with a stronger association between peer rejection and sensitivity than between parent rejection and sensitivity. Regarding interactive effects, peer rejection was found to have a strong association with rejection sensitivity among participants with low or high parent acceptance, and among those with high friendship satisfaction. Yet, there was evidence of a stronger association between peer rejection and rejection sensitivity among those with low parent acceptance or high friendship quality. This was because rejection sensitivity was highest when peer rejection was high and parent acceptance was low, and sensitivity was lowest when peer rejection was low and friendship quality was high. Findings show how young people's relationships in different domains uniquely covary with rejection sensitivity and interact in accounting for angry and anxious expectations of rejection by others.
\end{abstract}

Keywords: peer rejection, rejection sensitivity, parent-child relationships 


\section{Rejection Sensitivity in Childhood and Early Adolescence: Peer Rejection and Protective Effects of Parents and Friends}

Close relationships and social groups are important for biological and psychological security and well-being (Baumeister \& Leary, 1995) and rejection is particularly aversive and distressing (Leary, 2001). For children and adolescents, parent support is one important context of security that has been associated with many positive outcomes, such as high academic achievement and self-esteem (Doyle \& Markiewicz, 2005; Maccoby, 1980; Milevsky, Schlechter, Netter, \& Keehn, 2007), whereas many adjustment problems have been associated with rejecting or hostile parenting behaviours (e.g., Chang, Schwartz, Dodge, \& McBrideChang, 2003; Khaleque \& Rohner, 2002). Relationships with peers can also be quite important. During middle childhood, intimacy and companionship increases with peers, and children increasingly rely on peers for a sense of belonging (Laursen, 1996). At this time of life, peer relationship difficulties such as rejection and exclusion have been associated with internalising problems including loneliness and depression (Crick \& Grotpeter, 1995; Zimmer-Gembeck, Hunter, \& Pronk, 2007).

Young people's conceptions of relationships and their developing interpretations and responses to others have been proposed as some of the mechanisms accounting for associations between such negative social experiences and personal adjustment. For example, when rejection occurs within valued relationships with others, such as by parents or among peers at school, this can affect individuals' conceptions of relationships and their expectations of others, especially during childhood. Some of these conceptions have been referred to as rejection sensitivity, which is usually defined as anxious expectations of rejection and the tendency to readily perceive and overreact to it (Downey \& Feldman, 1996). When considered from this social cognitive perspective, rejection sensitivity identifies the types of social cues that will 
receive attention and the likelihood that an interpersonal interaction will be perceived as a "rejection" (Downey, Khouri, \& Feldman, 1997).

Most research on rejection sensitivity has focused on the negative correlates of being highly sensitive to rejection in later adolescence or adulthood, such as depressive affect and aggression. Rejection sensitivity has been shown to have negative consequences over and above the impact of actual rejection experiences (e.g., Sandstrom, Cillessen, \& Eisenhower, 2003), including impacting on loneliness, depression, aggression, dating violence, and relationship breakdown (Ayduk, Downey, \& Kim, 2001; Downey, Feldman, \& Ayduk, 2000; Levy, Ayduk, \& Downey, 2001; Zimmer-Gembeck \& Wright, 2007). However, an important aspect of rejection sensitivity theory suggests that acute and/or prolonged rejection experiences instigate rejection sensitivity (e.g., see Downey, Bonica, \& Rincon, 1999). Research on social experiences that may give rise to individual differences in rejection sensitivity or may protect against it, however, has lagged behind research on the negative mental health and socialrelational outcomes of sensitivity.

The purpose of the current study was to examine child and early adolescents' rejection sensitivity as related to parental rejection, peer rejection, parental acceptance, and friendship satisfaction. One particular focus was on examining interactions between experiences in different social-relational domains to test whether good relationships in one domain (e.g., acceptance by parents or a satisfying friendship) may protect against sensitivity in the face of rejection in another domain. This study also is unique because of its inclusion of children and early adolescents (ages 9 to 13 years), a time of life when peer relationships are becoming very important (Laursen, 1996).

\section{Social-relational Experiences during Early Adolescence and Rejection Sensitivity}

Theory emerged in the 1990 s to suggest that rejection sensitivity is a correlate of experiences of low acceptance and/or rejection by others in childhood (e.g., Downey et al., 
1999). In this theory, Downey and colleagues drew on attachment theory within an ecological framework and proposed that sensitivity to rejection is internalised from early experiences of rejection from others (see also Levy et al., 2001). Furthermore, utilising a social cognitive framework, rejection sensitivity was described as one process that follows from early experiences and affects the processing of social information in current and future situations (Feldman \& Downey, 1994). Therefore, rejection sensitivity is described as a process that mediates the link between early relational experiences and responses to current situations (Feldman \& Downey). More specifically, Feldman and Downey identified a history of parental and peer rejection and the combination of the two as the potential precursors to rejection sensitivity. When this is considered within a social cognitive approach, it is expected that rejection sensitivity shapes coding strategies, expectations, values and self regulatory plans that individuals bring to new situations.

Some research has examined associations between parent-child relationships and rejection sensitivity. In one of the early studies testing the theoretical underpinnings of rejection sensitivity, adults who retrospectively reported childhood violence were found to be more likely to anxiously expect rejection in adulthood than those who were not exposed (Feldman \& Downey, 1994). In another study, higher levels of parental neglect during childhood were associated with more rejection sensitivity in adulthood (Downey et al., 1997). Yet, because of the reliance on retrospective reports of parent-child relationships, this leaves only one previous study that has investigated contemporaneous associations between children or early adolescents' experiences of rejection by parents and rejection sensitivity. In this study, children who reported more parental rejecting behaviours responded to vignettes with more angry expectations of rejection from their peers and teachers over the following year (Downey, Lebolt, \& Rincon, 1995). 
We located only three previous investigations of associations between peer relationships and rejection sensitivity (or interpersonal sensitivity more generally). In one cross-sectional study, retrospective reports of teasing during childhood were associated with college students' interpersonal sensitivity (Butler, Doherty, \& Potter, 2007). In another study, no direct relationship was found between contemporaneous peer ratings of children's acceptance and rejection, on the one hand, and rejection sensitivity on the other hand, but self-reported levels of social acceptance and rejection did correlate with higher levels of some components of rejection sensitivity (Duzman, 2005). In the third study, being rejected increased boys' anxious and angry expectations of rejection, whereas acceptance was associated with fewer anxious expectations of rejection in boys and girls (London, Downey, Bonica, \& Paltin, 2007).

Strengths of these recent studies are the emergent focus on acceptance as well as rejection, and an acknowledgement that gender may also play a role. However, no previous study has simultaneously examined rejection sensitivity as a correlate of boys' compared to girls' relationships with peers and parents, as well as with a close friendship, which can be quite different than general acceptance or rejection by the larger peer group (Parker, Saxton, Asher, \& Kovacs, 1999). In addition, there has been no previous examination of the possibility that positive relationships in one social-relational domain (e.g., parents or peers) might buffer the negative experiences in another. There is, however, a long history of research supporting links between relationships and aspects of psychological well-being other than rejection sensitivity. Therefore, this research guided hypotheses about how multiple forms of relationship experiences would be simultaneously and jointly associated with rejection sensitivity. For example, parental support has been shown to be one of the three reoccurring themes in the lives of resilient children, showing that family support can buffer (i.e., moderate) negative outcomes from many forms of stress such as poverty and prejudice (Garmezy, 1983). In another study, Patterson, Cohn, and Kao (1989) studied 81, six-year-old children and their 
mothers to assess the protective factor of maternal warmth against peer rejection. The main finding was that rejected children whose mothers were rated as warm and accepting had fewer behavioural problems than rejected children whose mothers were low in warmth. This protective factor was more evident in more rejected compared to less rejected children.

With regards to friendship, satisfying friendships can also serve a protective function against rejection (e.g., Bollmer et al., 2005; Schmidt \& Bagwell, 2007). Therefore it was predicted that friendship quality would protect against peer group problems (i.e., rejection and exclusion). Furthermore, this research in conjunction with research that has found peer attachment to be just as important in childhood as parental attachment (e.g., Cotterell, 1992) indicated that friendship quality could also provide a protective function against parental rejection.

In summary, there were four specific aims in this study. Taken together, the aims addressed the roles of parents, peers, friends, and gender in early adolescents' rejection sensitivity. The first was to determine if rejection sensitivity is independently associated with rejection by parents, rejection by peers, acceptance by parents, and friendship satisfaction. The second aim was to test the buffering roles of parental acceptance and a satisfying friendship on the association between rejection sensitivity and peer rejection. The third aim was to determine if having a satisfying friendship could protect against rejection sensitivity in the face of parent rejection. The fourth and final aim was to examine gender differences in both levels of rejection sensitivity and associations between sensitivity and relationship experiences. Three hypotheses were tested. No hypotheses were made about gender. Instead, gender was explored as a potential moderator of associations found when testing these three hypotheses.

1. Parental and peer rejection will each be positively associated, and parent acceptance and friendship satisfaction will each be negatively associated with rejection sensitivity. 
2. Parental acceptance and friendship satisfaction will buffer the association between peer rejection and rejection sensitivity; the association between rejection and sensitivity will be weaker when parent acceptance is high rather than lower and when friendship satisfaction is high rather than lower.

3. Friendship satisfaction will buffer the association between parental rejection and rejection sensitivity; the association between rejection and sensitivity will be weaker when friendship satisfaction is high rather than lower.

\section{Method}

\section{Participants}

The study participants were 417 children and early adolescents from two large, Australian public schools. Schools contained children in grades 1 to 7, and all children in grades 5 to 7 were eligible to participate. Age ranged from 9 to $13(M=10.7, S D=1.1)$, with $53 \%$ girls and $47 \%$ boys. The schools included children with low to middle socioeconomic status and reflect the racial/ethnic diversity of the area; $80 \%$ were white/Caucasian with the other 20\% Asian, Indian, Middle Eastern, Aboriginal or Pacific Islander, or from a range of other sociocultural backgrounds. Of the parental consent forms distributed, $79 \%$ were returned and, of these, $87 \%$ agreed to participate, for a final participation rate of $65 \%$. The majority of children $(62 \%)$ lived with both of their biological parents. Students also assented to their own participation.

Measures

Rejection Sensitivity. The Children's Rejection Sensitivity Questionnaire (CRSQ; Downey et al., 1995; Downey, Lebolt, Rincon, \& Freitas, 1998) was used to measure anxious and angry expectations of rejection. The CRSQ consists of five vignettes involving peers and teachers (e.g., imagine you're in your classroom, and everyone is splitting up into groups to work on a special project together. You sit there and watch lots of other kids getting picked. As 
you wait, you wonder if the kids you want to be with will want you for their group). Following each vignette, children respond to three questions to gauge their affect and rejection expectations. The first two questions assessed anxious and angry responses by asking how nervous and how mad they would feel in this situation. Responses to these two items ranged from 1 (not at all) to 5 (yes/extremely). In the third question, children reported the likelihood of an accepting versus a rejecting response. Responses ranged from 1 (no) to 5 (yes).

Scoring of the CRSQ depends on weighting children's expectation of acceptance versus rejection by their anxious and angry responses. Two scores for each vignette are calculated by reversing the response to the expectation item before multiplying this response by children's responses regarding anxiety and anger. Responses are then summed to produce "cross situational" anxiety and anger scores. Finally, scores across the five vignettes are averaged to provide a total rejection sensitivity score. Higher scores indicated higher rejection sensitivity. Only the total rejection sensitivity score was used in the current study, Cronbach's $\alpha=.83$.

Parental acceptance and rejection. The acceptance and rejection subscales from the Parents as Social Context Questionnaire (PSCQ; Skinner, Johnson, \& Snyder, 2005) were used to measure children's perceived parental acceptance and rejection. Acceptance was defined as the expression of affection, love and kindness towards a child demonstrated by support and emotional availability. Rejection was defined as the expression of hostility, harshness and irritability towards a child demonstrated by criticism and disapproval.

Each subscale had 4 items. Sample items from the acceptance and rejection subscales are "my parents let me know they love me", and "sometimes I wonder if my parents like me", respectively. Responses to each item ranged from 1 (not at all true) to 5 (very true). Summary acceptance and rejection scores were obtained by averaging items on each subscale. In the current study, Cronbach's $\alpha=.82$ and $\alpha=.78$ for the acceptance and rejection subscales, respectively. 
Rejection by peers. Items from the Perception of Peers and Self Questionnaire (POPS; Rudolph, Hammen, \& Burge, 1995) were used to measure perceptions of peer rejection and exclusion. Items were either negatively worded, such as "friends often leave you out when there are other kids around to play with", or positively worded, such as "friends usually stick up for you when you're in trouble". Response options ranged from 1 (not at all true) to 5 (very true). After reversing positively worded items, the 15 items were averaged to assess rejection. In the current study, the Cronbach's $\alpha$ was .84.

Friendship satisfaction. Three items from the Network of Relationships Inventory (NRI; Furman \& Buhrmester, 1985) assessed children's satisfaction with their best friendship. An example item is "I think my best friendship is very good." Three items were used rather than the full scale because of school time constraints. Response options ranged from 1 (not at all true) to 5 (very true). Responses were averaged so that higher scores indicated more satisfaction with friendships. The three items yielded a Cronbach's $\alpha=.80$.

\section{Procedure}

Following ethical approvals, parental consent and child assent, students completed questionnaires in their classrooms. Instructions and questions were read aloud while children completed each question. Completion of the questionnaire took approximately 30 minutes.

\section{Results}

\section{Simple Correlations and Unique Associations}

Correlations. As expected, participants who reported more parental rejection also reported more rejection sensitivity in response to vignettes, and those who reported more parental acceptance were lower in rejection sensitivity (see Table 1). Also as anticipated, participants who reported more peer rejection reported more rejection sensitivity, and those who were more satisfied with their best friendships were lower in rejection sensitivity. 
Insert Table 1 here

Unique associations. When rejection sensitivity was regressed on measures of parental rejection, peer rejection, parental acceptance, and friendship satisfaction, a significant $34 \%$ of the variance was accounted for in rejection sensitivity, $F(6,410)=34.8, p<.01$. Parent and peer rejection were uniquely associated with more rejection sensitivity, but there were no significant associations of parental acceptance or friendship satisfaction with rejection sensitivity (see Table 2). A $z$-test determined that the association between peer rejection and rejection sensitivity was significantly stronger than the association between parental rejection and rejection sensitivity, $z=-7.95, p<.01$. Additionally, there was a small negative association between age and rejection sensitivity.

Insert Table 2 here

\section{Moderating Effects of Parental Acceptance and Friendship Satisfaction}

Both parental acceptance and friendship satisfaction were expected to buffer (reduce) the strength of the positive association between peer rejection and rejection sensitivity. Also, friendship satisfaction was expected to buffer the positive association between parental rejection and rejection sensitivity. Hence, three interactions were tested in three hierarchical regression models. The interactions were between 1) peer rejection and parent acceptance, 2) peer rejection and friendship satisfaction, and 3) parental rejection and friendship satisfaction. In each model, centred variables were used (see Jaccard, Turrisi, \& Wan, 1990). These were obtained by subtracting the mean of each variable from each participant's actual score. 
Centreing of variables is one suggested strategy for reducing the likelihood of multicollinearity concerns in multiple regression when testing interaction effects.

Parental acceptance buffering the association between peer rejection and rejection sensitivity. In Step 1 of the first model, rejection sensitivity was regressed on centred peer rejection and parental acceptance (see Model 1 in Table 3). To investigate whether parental acceptance buffered the association between peer rejection and rejection sensitivity, the interaction between parental acceptance and peer rejection was entered in Step 2 of the hierarchical regression model. The interaction term was significantly associated with rejection sensitivity, $\beta=-.14, p<.01, \Delta R^{2}=.016$. When this interaction effect was examined further, using techniques described by Jaccard et al. (1990), the association between peer rejection and rejection sensitivity was found to be weaker for participants who reported high rather than lower parental acceptance (see Figure 1a). It also revealed that rejection sensitivity was highest among young people who reported both high rejection by peers and low parental acceptance.

Insert Table 3 here

Insert Figure 1 (1a and 1b) here

To examine this finding further, we reanalysed these data replacing the measure of peer rejection with the sum of two items that participants completed to reported how much they thought the other kids in their grade and in their class liked them; each item had responses from 1 (not at all) to 5 (very much; metaperception, Cillessen \& Bukowski, 2000; Sandstrom et al., 2003). To form the interaction, this summary score was reversed and centred before forming the product term with parental acceptance. The reversed metaperception item was correlated with the POPS measure of peer rejection, $r=.58, p<.01$. Model 2 in Table 3 and Figure $1 \mathrm{~b}$ show the results of this analysis of metaperception. 
Taken together Figures $1 \mathrm{a}$ and $1 \mathrm{~b}$ show that, as expected, parental acceptance buffered the positive association between peer rejection and rejection sensitivity. This was true when peer rejection was measured with the POPS (Figure 1a) and when peer rejection was measured by metaperception (Figure 1b). Moreover, in both cases, young people reported the most rejection sensitivity when peer rejection was high and parental acceptance was low.

Friendship satisfaction buffering the association between peer rejection and rejection sensitivity. Rejection sensitivity was regressed on centred peer rejection and friendship satisfaction to test the hypothesised buffering effect of friendship satisfaction (see Model 1 in Table 4). In this analysis, one participant was found to be an extreme multivariate outlier. After removal of this participant, a significant moderating effect of friendship satisfaction was found, $\beta=.29, p<.05$, as expected. When this interaction was examined further, peer rejection was only a significant positive correlate of rejection sensitivity among those with high friendship satisfaction. In particular, it was children who reported low peer rejection and high friendship satisfaction that had the lowest rejection sensitivity. Nevertheless, this finding was fairly weak and was found only with one of our measures of peer problems; when these analyses were repeated replacing peer rejection with the measure of children's metaperception of being disliked by peers, there was no buffering effect of friendship quality (see Model 2 in Table 4).

\author{
Insert Table 4 here \\ Insert Figure 2 here
}

Friendship satisfaction buffering the association between parental rejection and rejection sensitivity. We estimated one final model to test the possibility that friendship satisfaction would buffer the negative association between parental rejection and rejection sensitivity. This hypothesis was not supported; the interaction was not significant . 


\section{Gender}

Gender differences in mean level. No difference in rejection sensitivity was found when boys and girls were compared. Boys did report more peer rejection than girls, $t(1,415)=3.15$, $p<.01$, whereas girls reported more friendship satisfaction than boys, $t(1.415)=-2.84, p<.01$. There were no gender differences in parental acceptance or rejection.

Gender moderation. We examined whether there were gender differences in associations of rejection sensitivity with parent and peer rejection, parent acceptance, and friendship satisfaction. This involved testing four product terms between 1) centred measures of parent and peer relationships and 2) gender. No interaction effects were significant, $\beta$ ranged from .08 to .04 . Associations of rejection sensitivity with parent and peer relationships did not seem to differ in boys compared to girls.

\section{Discussion}

The current research findings provide support for Downey and colleagues (Downey et al., 1999; Levy et al., 2001) model of rejection sensitivity, in which they proposed that both parental and peer rejection contribute to individual differences in rejection sensitivity during childhood and adolescence. The findings also provide preliminary evidence of an interactive effect between parent-child and peer relationships in relation to rejection sensitivity, and provide a foundation for considering how one relational domain, in this case peer group relationships, may be prominent in its implications for negative beliefs and expectations of rejection but that other domains, such as parent-child relationships and friendship, may also have negative or positive implications for rejection sensitivity when considered along with peer rejection.

In particular, hypotheses were supported regarding parent and peer rejection experiences as correlates of early adolescent's angry and anxious expectations of rejection (rejection sensitivity). These findings are consistent with previous research demonstrating the links 
between accounts of rejection-related experiences with parents and rejection sensitivity (Feldman \& Downey, 1994; Downey et al., 1995; Downey et al., 1997) and the link between peer rejection and heightened rejection sensitivity in children (Duzman, 2005; London et al., 2007). However, none of these previous studies focused on both parent and peer rejection simultaneously. Hence, this is the first study to show that parent and peer rejection each uniquely contribute to rejection sensitivity during middle/late childhood and early adolescence. This was expected, but we did not anticipate the very prominent role of peer rejection in rejection sensitivity, especially given that our participants were at an age when parents remain significant sources of support (Skinner \& Zimmer-Gembeck, 2007). Through all the analyses, it was clear that peer group rejection had the strongest association with rejection sensitivity. This finding illustrates the importance of the general peer group for early adolescents' expectations of others and their emotional reactions to perceived rejection.

This prominent role of peer rejection continued to be clear when parental acceptance and friendship satisfaction were examined as buffers (i.e., moderators) of the association between peer rejection and rejection sensitivity. More specifically, the positive relationship between peer rejection and rejection sensitivity was weaker when parental acceptance was high compared to low showing a protective influence of parent acceptance, as anticipated. This result is consistent with findings that parental acceptance protects against negative social expectations and hostile attributional biases (Liu, 2006; Steele, Steele, \& Johansson, 2002). Although there are multiple possible reasons why parents can play a protective role in the face of peer rejection, we expect that parents may be important because they help and guide their rejected children via discussing and explaining peer rejection in ways that impacts their understanding and minimises attributions of these experiences as internal (self-related) and stable patterns (Patterson, Cohn, \& Kao, 1989). 
What was also interesting were the significant interaction results found for 1) parent acceptance when a second measure of peer rejection, children's metaperceptions of how much their peers like them, was examined, and 2) the role of friendship satisfaction in the association between peer rejection and rejection sensitivity. Similar to parent acceptance, friendship satisfaction was expected to accompany reduced rejection sensitivity and was expected to moderate the association between peer rejection and rejection sensitivity. In both of these cases, good relationships with parents or friends did moderate the association between peer rejection and rejection sensitivity. Nevertheless, the buffering effect was somewhat different than anticipated for friendship satisfaction. Rather than showing that high friendship satisfaction can temper the association between peer rejection and rejection sensitivity, it showed that friendship satisfaction is associated with low rejection sensitivity especially when participants also report low peer rejection. Overall, peer group rejection appears to have such a prominent role in rejection sensitivity that when it is perceived to be high, children's anticipation, anxiety and anger about possible rejection events is high regardless of a good relationship with a friend. In addition, a satisfying friendship adds protective benefits for those who report that their peers generally like them. To our knowledge, no previous study has reported this prominent, overarching role for peer rejection, but also illustrates that we cannot ignore the role of good family and friend relationships in reducing rejection sensitivity.

Our final hypothesis was that friendship satisfaction would buffer the relationship between parental rejection and rejection sensitivity. This hypothesis was not supported. The parent's role in middle childhood has been described as one of giving, caring, helping and guiding, whereas peer relationships have been described as providing companionship and loyalty (Furman \& Buhrmester, 1985). Therefore, it is possible that a satisfying friendship with a peer does not have the provisions required to buffer rejection sensitivity in the face of rejection by parents, but it also may be that parents play a more minor role in the development 
of rejection sensitivity overall so that such a buffering influence of friendship is quite difficult to detect.

All of these findings should be considered along with some study limitations. In particular, the primary study limitations pertain to the reliance on child self-report only and the use of a cross-sectional study design. First, using only self-report measures may have inflated the strength of associations between variables. Yet, we believe it is important to obtain personal perceptions of relationships, and the use of vignettes to measure rejection sensitivity was expected to reduce shared method variance. Nevertheless, it is important to acknowledge that research has found perceptions of liking by peers to differ from peer reports of classmates who are liked or disliked (Zimmer-Gembeck, Hunter, \& Pronk, 2007). We did, however, have two measures of peer rejection which both yielded significant interaction effects when crossed with parent acceptance. Second, the use of a cross-sectional design limits the conclusions that can be drawn about directions of effects; it is possible that it is rejection sensitivity that creates rejection and lower acceptance for some children, as well as social experiences underpinning sensitivity (e.g., Purdie \& Downey, 2000). However, our findings in such a large sample and with a young age group are important evidence to support future longitudinal research to extend these findings.

In summary, the current study has implications for rejection sensitivity theory and intervention. In relation to theory, the findings provide the first evidence for the unique roles of peer and parental rejection in accounting for rejection sensitivity. Another extension on rejection sensitivity theory is suggested by the finding that peer rejection may be a greater risk factor for rejection sensitivity than parental rejection, but that parents can buffer the negative impact of peer rejection on their children's angry and anxious expectations of rejection by others. More research is needed that examines the interactions between parental and peer relationships or general peer relationships and close friendships. Such interactive effects have 
not been considered in previous research into child or adolescents' views and expectations of relationships, but these findings show that relationships with others may work together to impact upon understanding of others and perceptions of others' behaviours. Such evidence could guide the design of interventions by suggesting that children and early adolescents may benefit from the involvement of parents, especially when they are rejected by their peers, and that children do accrue benefits from both being generally accepted by their peers and having satisfying close friendships. 


\section{References}

Ayduk, O., Downey, G., \& Kim, M. (2001). Rejection sensitivity and depressive symptoms in women. Personality and Social Psychology Bulletin, 27, 868-877.

Baumeister, R. M., \& Leary, M. R. (1995). The need to belong: Desire for interpersonal attachments as fundamental human motivation. Psychological Bulletin, 117, 497-529.

Butler, J. C., Doherty, M. S., \& Potter, R. M. (2007). Social antecedents and consequences of interpersonal rejection sensitivity. Personality and Individual Differences, 43, 13761385 .

Chang, L., Schwartz, D., Dodge, K. A., \& McBride-Chang, C. (2003). Harsh parenting in relation to child emotion regulation and aggression. Journal of Family Psychology, 17, 598-606.

Cillessen, A. H. N., \& Bukowski, W. M. (Eds.). (2000). Recent advances in the measurement of acceptance and rejection in the peer system. San Francisco: Jossey-Bass.

Downey, G., Bonica, C., \& Rincon, C. (1999). Rejection sensitivity and adolescent romantic relationships. In W. Furman, B. Bradford Brown, \& C. Feiring (Eds.), The development of romantic relationships in adolescence (pp. 148-174). Cambridge: Cambridge University Press.

Downey, G., \& Feldman, S. I. (1996). Implications of rejection sensitivity for intimate relationships. Journal of Personality and Social Psychology, 70, 1327-1343.

Downey, G., Feldman, S., \& Ayduk, O. (2000). Rejection sensitivity and male violence in romantic relationships. Personal Relationships, 7, 45-61.

Downey, G., Khouri, H., \& Feldman, S. (1997). Early interpersonal trauma and later adjustment: The meditational role of rejection sensitivity. In D. Cicchetti \& S. Toth (Eds.), Rochester symposium on developmental psychopathology: Theory, research and intervention (pp. 85-114). Rochester, New York: University of Rochester Press. 
Downey, D., Lebolt, A., \& Rincon, C. (1995). The development of a measure of rejection sensitivity for children. Unpublished manuscript.

Downey, G., Lebolt, A., Rincon, C., \& Freitas, A. L. (1998). Rejection sensitivity and children's interpersonal difficulties. Child Development, 69, 1074-1091.

Doyle, A. B., \& Markiewicz, D. (2005). Parenting, marital conflict and adjustment from earlyto mid-adolescence: Mediated by adolescent attachment style? Journal of Youth and Adolescence, 34, 97-110.

Duzman, A. M. (2005). The relationship between rejection sensitivity, peer social standing, and perceived social acceptance among pre-adolescents. Unpublished dissertation, Alliant International University, California, America (UMI No. 3166040).

Feldman, S., \& Downey, G. (1994). Rejection sensitivity as a mediator of the impact of childhood exposure to family violence on adult attachment behavior. Developmental and Psychopathology, 6, 231-247.

Furman, W., \& Buhrmester, D. (1985). Children's perceptions of the personal relationship in their social networks. Developmental Psychology, 21, 1016-1024.

Harter, S. (1988). Manual for the self-perception profile for adolescence. Denver: University of Denver.

Hymel, S., \& Franke, S. (1985). Children's peer relations: Assessing self-perceptions. In B. H. Schneider, K. H. Rubin, \& J. E. Ledingham (Eds.), Children's peer relations: Issues in assessment and intervention (pp. 75-91). New York: Springer-Verlag.

Jaccard, J., Turrisi, R., \& Wan, C. K. (1990). Interaction effects in multiple regression. California: Sage Publications.

Khaleque, A., \& Rohner, R. P. (2002). Perceived parental acceptance-rejection and psychological adjustment: A meta-analysis of cross-cultural and intracultural studies. Journal of Marriage and Family, 64, 54-64. 
Laursen, B. (1996). Closeness and conflict in adolescent peer relationships: Interdependence with friends and romantic partners. In W. M. Bukowski, A. F. Newcomb, \& W. W. Hartup (Eds.), The company they keep: Friendship in childhood and adolescence (pp. 186-210). New York: Cambridge University Press.

Leary, M. R. (2001). Toward a conceptualization of interpersonal rejection. In M. R. Leary (Ed.), Interpersonal rejection (pp. 3-20). New York: Oxford University Press.

Levy, S. R., Ayduk, O., \& Downey, G. (2001). The role of rejection sensitivity in people's relationships with significant others and valued social groups. In M. R. Leary (Ed.), Interpersonal rejection (pp. 251-289). New York: Oxford University Press.

Liu, Y. (2006). Paternal/maternal attachment, peer support, social expectations of peer interaction, and depressive symptoms. Adolescence, 41, 705-721.

London, B., Downey, G., Bonica, C., \& Paltin, I. (2007). Social causes and consequences of rejection sensitivity. Journal of Research on Adolescence, 17, 481-506.

Maccoby, E. E. (1980). Social development: Psychological growth and the parent-child relationship. New York: Harcourt Brace Jovanovich.

Milevsky, A., Schlechter, M., Netter, S., \& Keehn, D. (2007) Maternal and paternal parenting styles in adolescents: Associations with self-esteem, depression and life-satisfaction. Journal of Child and Family Studies, 16, 39-47,

Parker, J. G., Saxton, J. L., Asher, S. R., \& Kovacs, D. M. (1999). Dimensions of children's friendship adjustment: Implications for understanding loneliness. In K. J. Rotenberg \& S. Hymel (Eds.), Loneliness in childhood and adolescence (pp. 201-221). New York: Cambridge University Press.

Patterson, C., Cohn, D., \& Kao, B. (1989). Maternal warmth as a protective factor against risks associated with peer rejection among children. Development and Psychopathology, 1, 21-38. 
Purdie, V., \& Downey, G. (2000). Rejection sensitivity and adolescent girls' vulnerability to relationship-centred difficulties. Child Maltreatment, 5, 338-349.

Rudolph, K. D., Hammen, C., \& Burge, D. (1995). Cognitive representations of self, family, and peers in school-age children: Links with social competence and sociometric status. Child Development, 66, 1385-1402.

Sandstrom, M. J., Cillessen, A. H. N., \& Eisenhower, A. (2003). Children's appraisal of peer rejection experiences: Impact on social and emotional adjustment. Social Development, $12,530-550$.

Skinner, E., Johnson, S., \& Snyder, T. (2005). Six dimensions of parenting: A motivational model. Parenting: Science and Practice, 5, 175-235.

Skinner, E. A., \& Zimmer-Gembeck, M. J. (2007). The development of coping. Annual Review of Psychology, 58, 119-144.

Steele, M., Steele, H., \& Johansson, M. (2002). Maternal predictors of children's social cognition: an attachment perspective. Journal of Child Psychology and Psychiatry, 43, $861-872$.

Zimmer-Gembeck, M. J., Hunter, T. A., \& Pronk, R. (2007). A model of behaviors, peer relations and depression: Perceived social acceptance as a mediator and the divergence of perceptions. Journal of Social and Clinical Psychology, 26, 273-302.

Zimmer-Gembeck, M. J., \& Wright, J. L. (2007). Rejection sensitivity and romantic attachment orientation are associated with parental psychological control. In R. S. Grenell (Ed.), Adolescent behavior research studies (pp. 121-139). New York: NOVA Science Publishers. 
Table 1

Pearson's Correlations between Measures $(N=417)$

\begin{tabular}{|c|c|c|c|c|c|}
\hline Variables & 1 & 2 & 3 & 4 & 5 \\
\hline 1. Rejection sensitivity & -- & & & & \\
\hline 2. Parental rejection & $.36 * *$ & -- & & & \\
\hline 3. Peer rejection & $.56^{* *}$ & $.44 * *$ & -- & & \\
\hline 4. Parental acceptance & $-.24 * *$ & $-.57 * *$ & $-.34 * *$ & -- & \\
\hline 5. Friendship satisfaction & $-.30 * *$ & $-.26 * *$ & $-.57 * *$ & $.26^{* *}$ & -- \\
\hline Age & -.07 & .03 & .03 & -.04 & .01 \\
\hline$M(S D)$ & $6.2(3.0)$ & $1.8(0.9)$ & $2.3(0.7)$ & $4.6(0.7)$ & $4.6(0.8)$ \\
\hline
\end{tabular}


Table 2

Results of Regressing Rejection Sensitivity on all Measures $(N=417)$

\begin{tabular}{lrc} 
Variables & $B(S E)$ & $\beta$ \\
Age & $-.23(.11)$ & $-.08^{*}$ \\
Gender & $.25(.24)$ & .04 \\
Parental rejection & $1.47(.50)$ & $.15^{* *}$ \\
Peer rejection & $2.33(.24)$ & $.52^{* *}$ \\
Parental acceptance & $-.69(.93)$ & -.04 \\
Friendship satisfaction & $-.23(.85)$ & -.01 \\
\hline
\end{tabular}

$R^{2}=.34, F(6,410)=34.84, p<.01$.

${ }^{*} p<.05 .{ }^{* *} p<.01$. 
Table 3

Results of Testing Parental Acceptance as a Moderator of the Association between Peer Rejection and Rejection Sensitivity $(N=417)$

\begin{tabular}{|c|c|c|c|c|}
\hline \multirow[b]{2}{*}{ Variables } & \multicolumn{2}{|c|}{$\begin{array}{c}\text { Model 1, POPS Peer } \\
\text { Rejection }\end{array}$} & \multicolumn{2}{|c|}{$\begin{array}{l}\text { Model 2, Children's Meta- } \\
\text { perception of Peer Rejection }\end{array}$} \\
\hline & $B(S E)$ & $\beta$ & $B(S E)$ & $\beta$ \\
\hline \multicolumn{5}{|l|}{ Step 1} \\
\hline Age & $-.23(.11)$ & $-.08 *$ & $-.21(.13)$ & $-.08 *$ \\
\hline Gender & $.25(.24)$ & .04 & $-.02(.27)$ & .00 \\
\hline A - Peer rejection & $2.49(.20)$ & $.56^{* *}$ & $1.38(.18)$ & $.36^{* *}$ \\
\hline B - Parental acceptance & $-.52(.83)$ & -.03 & $-2.49(.89)$ & $-.13 * *$ \\
\hline \multicolumn{5}{|l|}{ Step 2} \\
\hline Age & $-.21(.11)$ & $-.08 *$ & $-.20(.13)$ & -.07 \\
\hline Gender & $.31(.24)$ & .05 & $-.07(.26)$ & -.01 \\
\hline $\mathrm{B}-$ Peer rejection & $2.45(.19)$ & $.55^{* *}$ & $1.28(.18)$ & $.33 * *$ \\
\hline A - Parental acceptance & $.60(.90)$ & .03 & $-1.61(.92)$ & -.08 \\
\hline$A \times B$ & $-3.12(.99)$ & $-.14 * *$ & $-2.93(.84)$ & $-.17 * *$ \\
\hline
\end{tabular}

Note. Model $1 R^{2}=.323$ for Step $1(F(4,412)=49.24, p<.01) ; \Delta R^{2}=.016$ for Step $2(\Delta F(1$, $411)=9.84, p<.01)$. Model $2 R^{2}=.174$ for Step $1(F(4,412)=121.74, p<.01) ; \Delta R^{2}=.024$ for Step $2(\Delta F(1,411)=12.25, p<.01)$.

${ }^{*} p<.05 .{ }^{* *} p<.01$. 
Table 4

Results of Testing Friendship Satisfaction as a Moderator of the Association between Peer Rejection and Rejection Sensitivity $(N=416)$

\begin{tabular}{lcccc}
\hline & \multicolumn{2}{c}{$\begin{array}{c}\text { Model 1, POPS Peer } \\
\text { Rejection }\end{array}$} & \multicolumn{2}{c}{$\begin{array}{c}\text { Model 2, Children's Meta- } \\
\text { perception of Peer Rejection }\end{array}$} \\
\hline Variables & $B(S E)$ & $\beta$ & $B(S E)$ & $\beta$ \\
Step 1 & $-.20(.11)$ & $-.08^{*}$ & $-.16(.12)$ & -.06 \\
Age & $.26(.24)$ & .04 & $.12(.26)$ & .02 \\
Gender & $2.43(.22)$ & $.57^{* *}$ & $1.10(.19)$ & $.29 * *$ \\
A - Peer rejection & $-.30(.84)$ & -.02 & $-3.24(.81)$ & $-.20^{* *}$ \\
B - Friendship satisfaction & & & $-.16(.12)$ & -.06 \\
Step 2 & $-.20(.11)$ & -.07 & $.12(.26)$ & .02 \\
Age & $.27(.24)$ & .05 & $1.14(.19)$ & $.30^{* *}$ \\
Gender & $1.16(.70)$ & $.27^{* *}$ & $-3.49(.86)$ & $-.21^{* *}$ \\
A - Peer rejection & $-.57(.95)$ & -.03 & $.13(.15)$ & .04 \\
B - Friendship satisfaction & $1.89(.89)$ & $.29^{*}$ & \\
A x B & & & & \\
\hline
\end{tabular}

Model 1: $R^{2}=.299$ for Step $1(F(4,411)=43.58, p<.01) ; \Delta R^{2}=.006$ for Step $2\left(F_{\text {chg }}(1,410)\right.$ $=3.62, p<.05)$.

Model 2: $R^{2}=.159$ for Step $1(F(4,411)=19.37, p<.01) ; \Delta R^{2}=.001$ for Step $2\left(F_{\text {chg }}(1,410)\right.$ $=.684, p=.41)$. $* p<.05 . * * p<.01$. 


\section{Figure Headings}

Figure 1. Illustrations of parental acceptance moderating the association between perceived peer rejection and rejection sensitivity (1a) and between metaperception and rejection sensitivity (1b).

Figure 2. Illustration of friendship satisfaction moderating the association between peer rejection and rejection sensitivity. 
Figure 1a

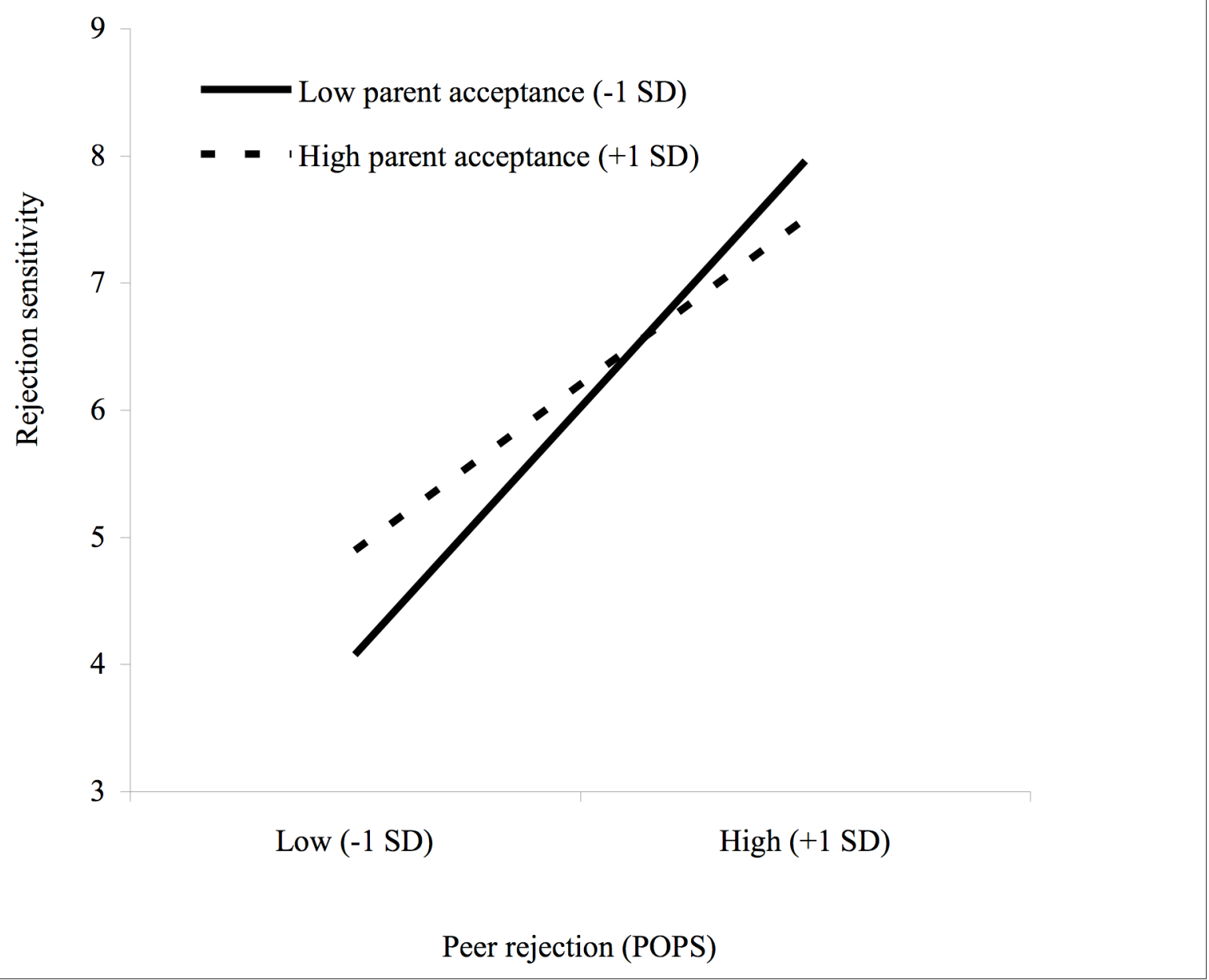


Figure 1b

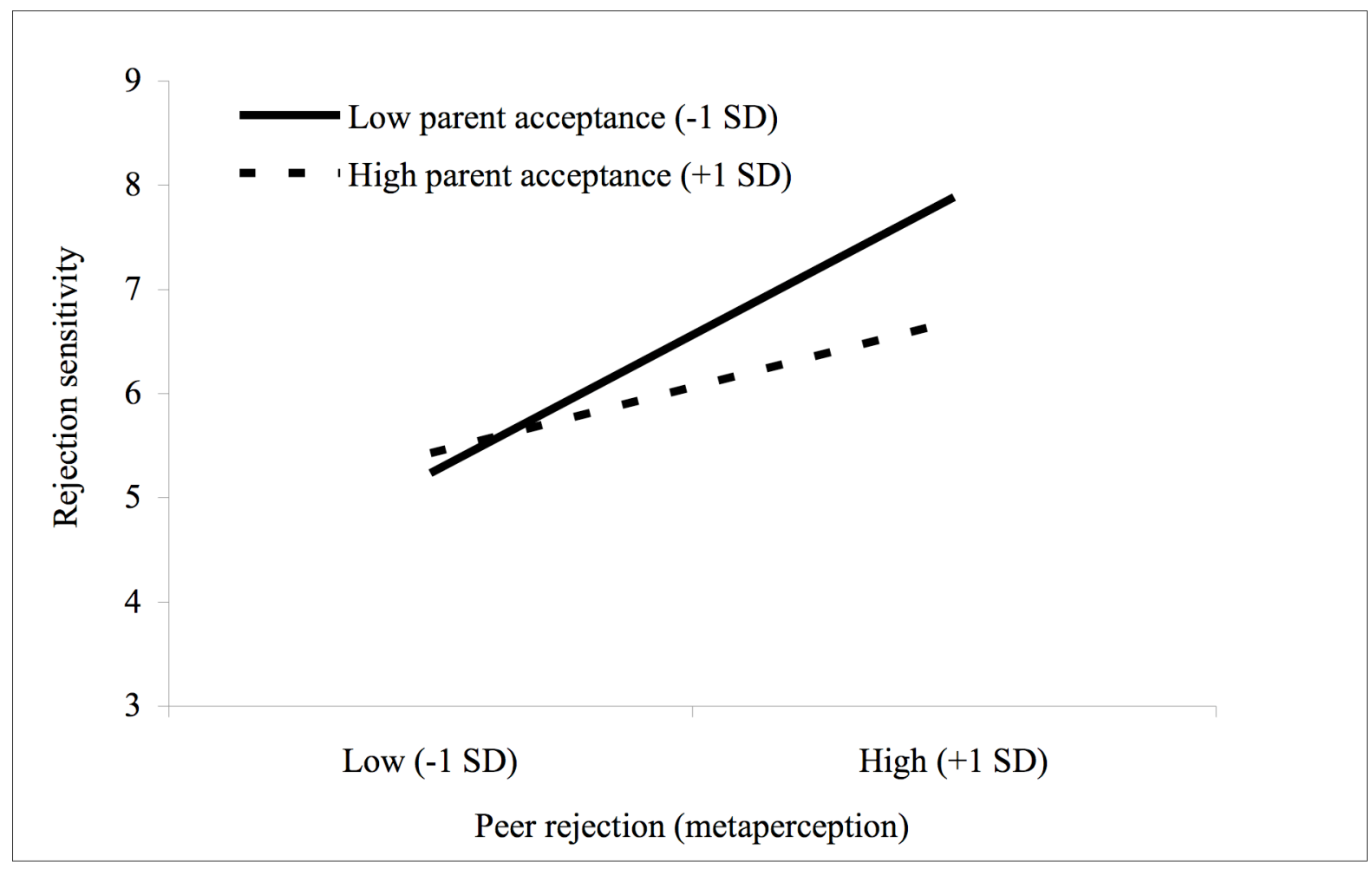


Figure 2

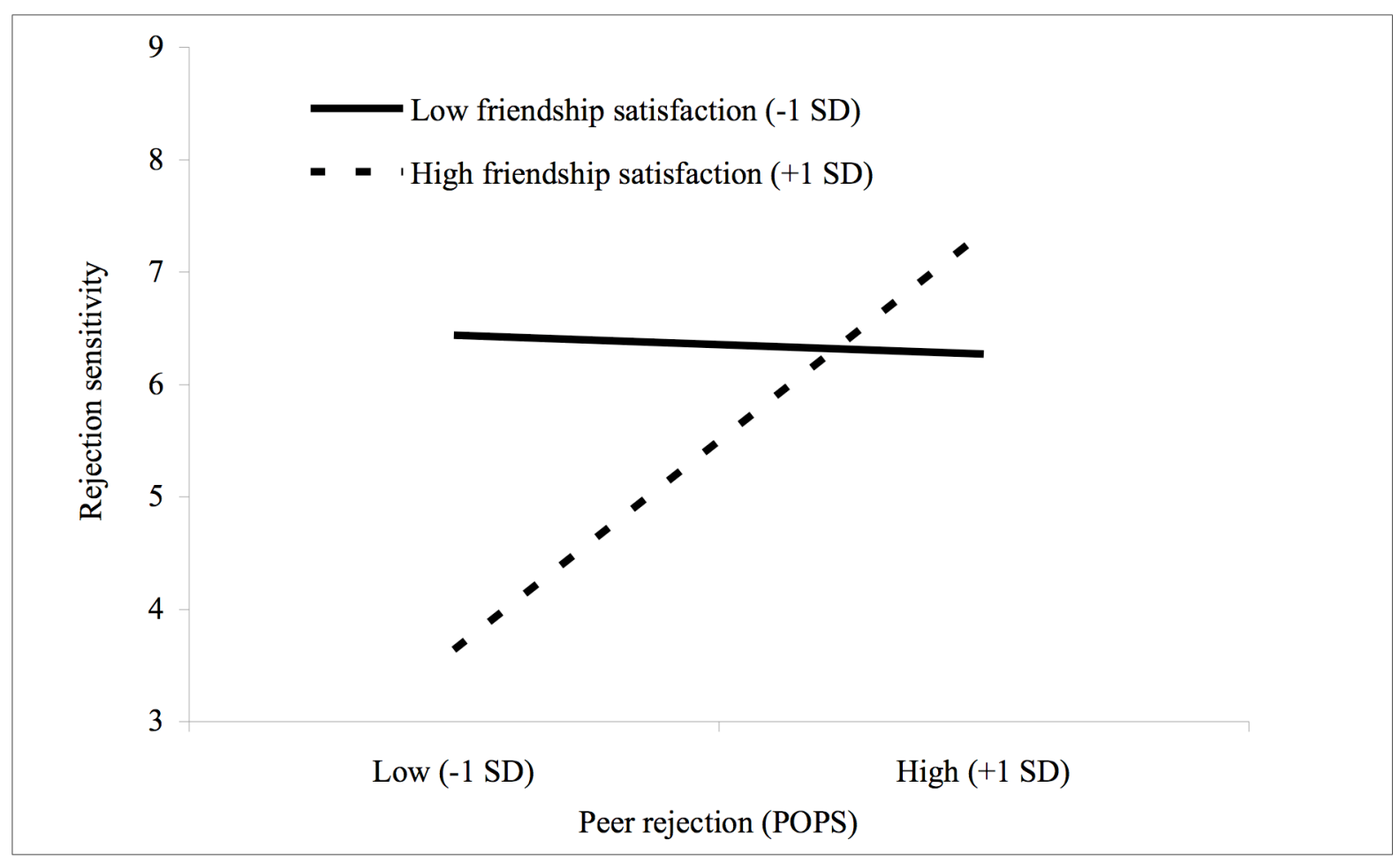

